\title{
Слоевое сопротивление TiAINiAu тонкопленочной металлизации омических контактов к нитридным полупроводниковым структурам
}

\author{
(C) H.A. Торхов ${ }^{1,2,3}$ \\ ${ }^{1}$ АО „Научно-исследовательский институт полупроводниковых приборов“, \\ 634034 Томск, Россия \\ ${ }^{2}$ Томский университет систем управления и радиоэлектроники, \\ 634050 Томск, Россия \\ ${ }^{3}$ Томский государственный университет, \\ 634050 Томск, Россия \\ E-mail: trkf@mail.ru
}

(Получена 10 апреля 2018 г. Принята к печати 23 апреля 2018 г.)

\begin{abstract}
Определена связь геометрии метрического пространства поверхности тонкопленочной металлической TiAlNiAu-системы с геометрией функционального пространства ее слоевых сопротивлений $R_{s q}$. На основе этого описан наблюдающийся в локальном приближении латеральный размерный эффект, который проявляется в зависимости слоевого сопротивления $R_{s q}$ металлической TiAlNiAu-пленки от ее латеральных (в плоскости $(x, y))$ линейных размеров. Зависимость $R_{s q}$ от линейных размеров определяется фрактальной геометрией образующих ее дендритов, а именно - степенной зависимостью изменения линейных размеров от фрактальной размерности $D_{f}$. Обнаруженная закономерность имеет важное практическое значение как для точного расчета значений $R_{s q}$ тонкопленочных металлических систем при проектировании дискретных приборов и интегральных микросхем, так и для контроля результатов технологических процессов получения тонких металлических пленок и систем на их основе на микронном и наноуровнях.
\end{abstract}

DOI: $10.21883 /$ FTP.2019.01.46983.8886

\section{1. Введение}

В большинстве случаев металлические пленки нанометровой толщины представляют собой совокупность расположенных на плоскости $(x, y)$ наноразмерных проводящих зерен или кристаллитов, разделенных между собой диэлектрическими промежутками - модель Маядаса-Шатцкеса [1]. Рост таких пленок происходит за счет разрастания и срастания зародышевых зерен или кристаллитов, сопровождающееся увеличением их толщины и проводящей способности [2-8]. Протекание или не протекание электричества через такие системы, представляющие собой чередование в плоскости $(x, y)$ проводящих и непроводящих локальных областей - явление перколяции, описывается теорией перколяции [9]. Большая часть работ в этом направлении была выполнена в предположении однородности электромагнитного поля внутри пленки [10,11]. В сантиметровом и миллиметровом частотных диапазонах для пленок толщиной 100-150 нм это условие плохо выполняется, что затрудняет применение известных физических моделей тонкопленочных систем $[1,4-6,9,12]$ и интерпретацию полученных результатов. При этом именно на эти частотные диапазоны электромагнитных колебаний в настоящее время и приходится основной объем по применению тонкопленочных металлических систем в омических и барьерных контактах, тонкопленочных резисторах, конденсаторах, линиях СВЧ-передачи, контактных площадках и т. п.

Исследования перколяционных эффектов в металлических пленках были связаны в основном с изучением влияния поперечных (перпендикулярно плоскости $(x, y))$ размеров (толщин $d)$ на их проводимость. Наблюдаемые в таких пленках изменения проводимости большинство исследователей связывало с особенностями рассеяния носителей заряда на границах образующих их зерен и кристаллитов [12], неровностях поверхности [13]. В рамках теории Фукса-Зондгеймера удельная проводимость тонких металлических пленок даже без учета такого рассеяния значительно отличается от удельной проводимости используемых при их изготовлении объемных материалов [4-6]. В частности, исследование строения пленок большей части используемых в электронной промышленности металлов (золота $\mathrm{Au}[14,15]$, молибдена Мо [16], вольфрама W [17], серебра Ag [18], висмута Вi [19,20], титана Ti $[21,22]$, меди $\mathrm{Cu}[23,24]$, цинка $\mathrm{Zn}[25,26]$, кобальта Co [27,28], платины Pt [29], палладия Pd [30], олова $\mathrm{Sn}[31]$, алюминия $\mathrm{Al}[32,33]$, никеля $\mathrm{Ni}$ [34-36], ниобия $\mathrm{Nb}[10,11]$, тантала Та $[10,11]$, хрома $\mathrm{Cr}[10,11]$, рения $\operatorname{Re}[10,11]$, скандия $\mathrm{Sc}[10,11]$ и др.) выявило сильную зависимость их проводимости и, в некоторых случаях, поведения малосигнальных параметров рассеяния высокочастотных сигналов от их поперечных размеров толщин $d[36-41]$.

Между тем стоит отметить, что большая часть тонкопленочных металлических систем в локальном приближении имеет фрактальную геометрию, например, [42,43-45], влияние которой на их электрофизические характеристики в зависимости от их латеральных размеров в плоскости $(x, y)$ - размерный эффект в настоящее время остается практически неизученным. Из-за этого разработка и изготовление тонкопленочных металлических систем с заданными характеристиками в 
настоящее время носит в основном поисковый итерационный характер.

Напомним, что в современном полупроводниковом материаловедении линейные размеры областей локального приближения $L$, в которых могут проявляться латеральные размерные эффекты, выходят за пределы нанометровых размеров и часто соизмеримы или даже превышают линейные размеры основных конструктивных элементов полупроводниковых приборов (десятки и сотни микрометров) [45-50]. В связи с этим для определения понятий размерных эффектов в данной работе будем исходить из потребностей планарных полупроводниковых технологий, где под линейными размерами понимаются размеры основных конструктивных элементов полупроводниковых приборов и латеральных (в плоскости $(x, y))$ неоднородностей: линейные размеры барьерных и омических контактов, тонкопленочных резисторов, конденсаторов, линий СВЧ-передач, неровностей рельефа $h(x, y)$, неоднородностей поверхностного потенциала $\Delta \varphi(x, y)$, фазового состава и т. п. $[45,46]$.

В частности, большой научно-практический интерес представляет связь геометрии металлической системы TiAlNiAu, нашедшей широкое применение в качестве металлизации низкоомных омических контактов (ОК) к нитриду галлия электронного типа проводимости $n^{+}-\mathrm{GaN}$, с ее электрофизическими свойствами $[43,46,51-53]$.

Таким образом, можно сформулировать задачу по исследованию размерных эффектов, проявляющихся в зависимости слоевых сопротивлений $R_{s q}$ многослойной металлической системы на основе TiAlNiAu от ее латеральных размеров.

\section{2. Методическая часть}

Для характеристики электрических сопротивлений тонких металлических пленок и систем на их основе, латеральные размеры которых в плоскости пленки $(x, y)$ значительно превышают их толщину, часто используют понятие слоевого сопротивления, определяемого как „сопротивление на квадрат“ $R_{s q}-$ сопротивление квадpaтных $R_{\square}$ (рис. $\left.1, a\right)$ или круглых $R_{\mathrm{O}}($ рис. $1, b)$ участков этих пленок.

В работе для исследования слоевого сопротивления $R_{s q}$ многослойной металлической системы TiAlNiAu $=50 \times 50 \times 150 \times 100$ нм, сформированной на поверхности полуизолирующего буферного слоя $i$-GaN, использовались круглые TiAlNiAu-контакты радиусами $r_{i}: r_{1}=20, \quad r_{2}=40, \quad r_{3}=100, \quad r_{4}=200 \quad$ и $r_{5}=500$ мкм - тесты контактов, полученные методами взрывной литографии и электронно-лучевого напыления (рис. 1). После этого тесты подвергались быстрому термическому отжигу в режиме, используемом для формирования омических контактов к нитридным НЕМТ-структурам при температуре $780^{\circ} \mathrm{C}$ в течение $30 \mathrm{c}$. Измеренная средняя толщина контактной металлизации после отжига составила $d=310 \pm 60$ нм.
В общем случае однородные по толщине $d(x, y)=$ $=$ const и удельному сопротивлению $\rho(x, y)=$ const $([\rho]=$ Ом $\cdot \mathrm{M})$ тонкие пленки обладают тем свойством,

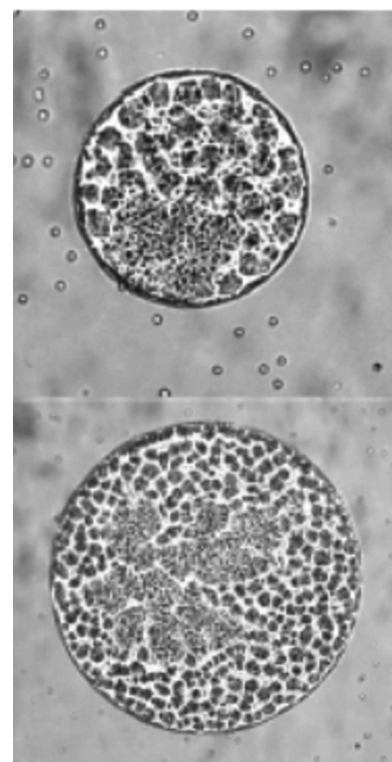

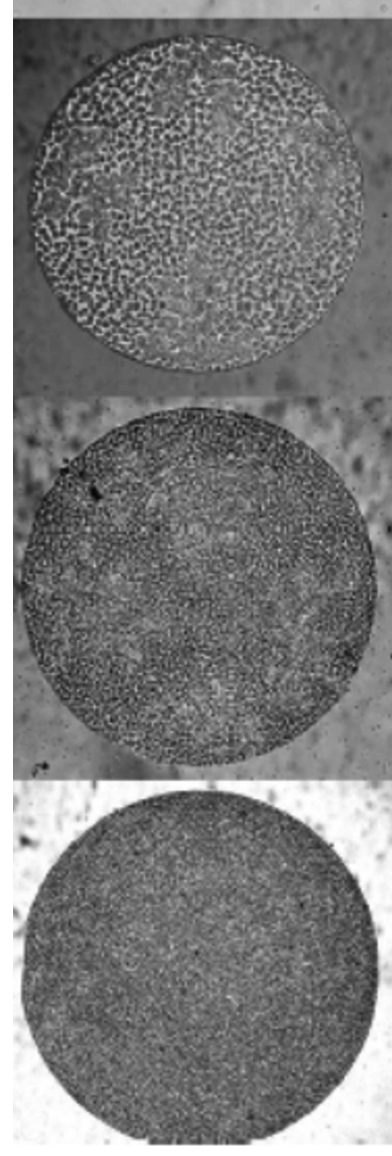

I

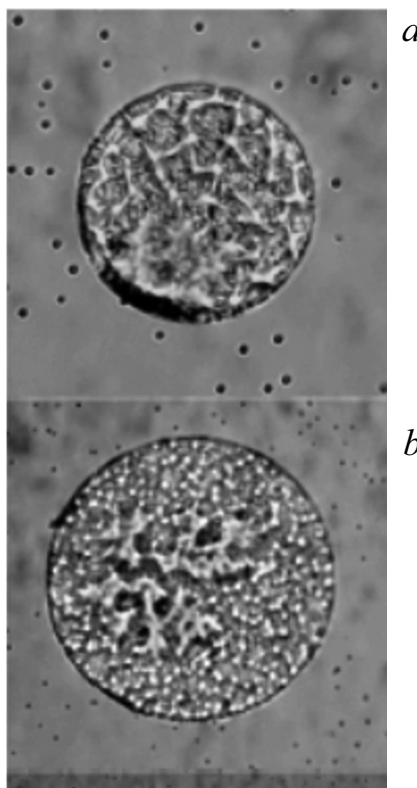

$c$

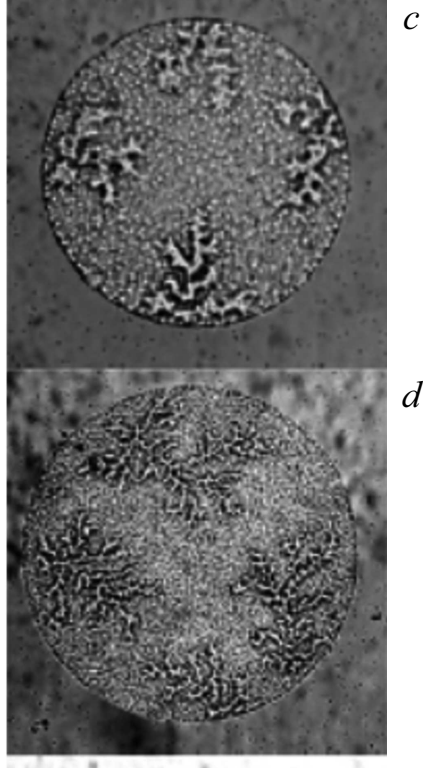

$e$

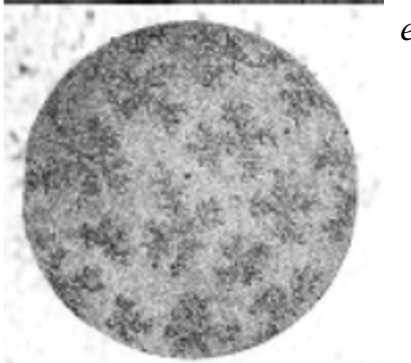

II
Рис. 1. Оптические изображения поверхностей TiAlNiAu тестовых контактов радиусами: $r_{1}=20(a), r_{2}=40(b)$, $r_{3}=100(c), r_{4}=200(d)$ и $r_{5}=500$ мкм $(e)$ при фокусировке на дне ячеистой структуры (I) и на поверхности дендритов (II). 

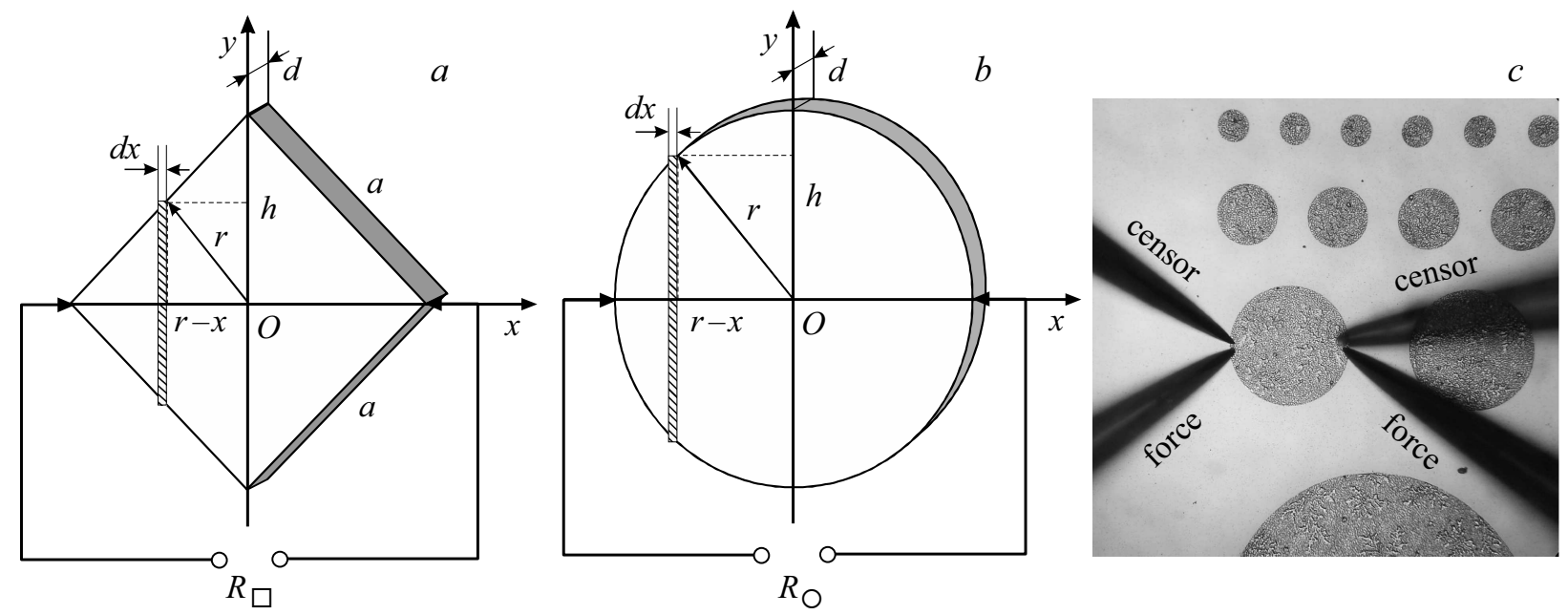

Рис. 2. Схемы расчета слоевых сопротивлений тонких однородных пленок толщиной $d$ : квадратных $(a)$, круглых $(b)$ и фотография, показывающая процесс измерения слоевого сопротивления четырехзондовым методом Кельвина $(c)$.

что слоевое сопротивление (латеральная проводимость) их квадратных $R_{\square}$ или круглых $R_{\mathrm{O}}$ участков не зависит от их линейных размеров: стороны квадратного участка a $\left(R_{\square}(a)=\right.$ const, рис. $\left.2, a\right)$ или радиуса $r$ $\left(R_{\mathrm{O}}(r)=\right.$ const, pис. $\left.2, b\right)$ :

$$
R_{\mathrm{O}}=2 \int_{0}^{r} \frac{\rho d x}{d 2 h}=\frac{\pi \rho}{2 d}=\frac{\pi}{2} R_{\square}, \quad R_{\square}=\frac{\rho}{d}=\text { const. }
$$

Контроль рельефа $h(x, y)$ броуновских поверхностей тестовых TiAlNiAu-контактов проводился методами оптической микроскопии.

Слоевое сопротивление тестов $R_{\mathrm{O}}$ (проводимость $G=1 / R_{\mathrm{O}}$ ) определялось измерителем характеристик полупроводниковых приборов $\mathrm{B}-1500$ производства „Keysight technologies“ на зондовой станции „М-150“ производства Cascade Microtech. Для исключения влияния последовательных сопротивлений, вносимых контактами измерительных зондов к поверхности тестов, использовался четырехзондовый метод Кельвина (рис. 2, c). Погрешность при измерении сопротивления в диапазоне смещений от -5 до +5 В не превышала 0.04 Ом.

Из общих соображений понятно, что геометрическая форма не может быть описана только с использованием одной размерности $D_{f}$. Поэтому для изучения особенностей геометрии исследуемых объектов необходимо использовать их геометрические модели. Моделирование фрактальной структуры кристаллитов осуществлялось с использованием метода внерешеточного моделирования многочастичным методом Монте-Карло. В модельных экспериментах использовался ансамбль из 30000 броуновских частиц. Алгоритм многочастичного метода Монте-Карло заключался в следующем. Модельные частицы ансамбля, за время $\tau$ поочередно из выбранных случайным образом точек непрозрачного замкнутого контура, совершали внутри этого контура в плоскости $(x, y)$ дискретные перемещения на $\Delta r$. В качестве такого контура выбирался круг (рис. 3, $a$, сплошная кривая). Затем отслеживались элементарные (скачкообразные) $\Delta r$ перемещения поочередно всех частиц за временной интервал $\tau$ и запоминались их положения. Далее процедура повторялась аналогичным образом в течение заданного количества циклов $N$. После анализа состояния снова делался шаг по времени $\tau$ и т.д. Таким образом, моделировалась временная эволюция системы в течение времени $t=\tau N$. Для моделирования движения частиц использовалась модель случайных блужданий.

Модельный кластер начинал расти из одной или нескольких расположенных внутри контура зародышевых точек - зародышей. Броуновские частицы ансамбля, соприкасаясь с растущим кластером, имели возможность прилипнуть к некоторым его областям с заданной вероятностью, определяющей разветвленность кластера. Разветвленность кластера определялась числом связей на единицу длины составляющих его самоподобных участков, т.е. значением скейлинговых коэффициентов $\eta$ и $\zeta$.

\section{3. Теоретическая часть}

Для исследования связи геометрии TiAlNiAu-тестов с их сопротивлением $R_{\mathrm{O}}$ использовались понятия меры, метрики, метрических и нормированных функциональных (линейных) пространств, позволяющие с единых позиций рассматривать скалярные и векторные величины, непрерывные функции и числовые последовательности.

Мера измеряемого объекта $M$ в общем случае представляет собой интеграл от некоторой функции, определенной на семействах множеств пространств Минковского, и должна обладать свойством аддитивности и удовлетворять аксиомам треугольника, симметрии и нулевого расстояния [54]. Пространство Минковского - четырехмерное псевдоевклидово пространство сигнатуры $\{1,3\}$. 

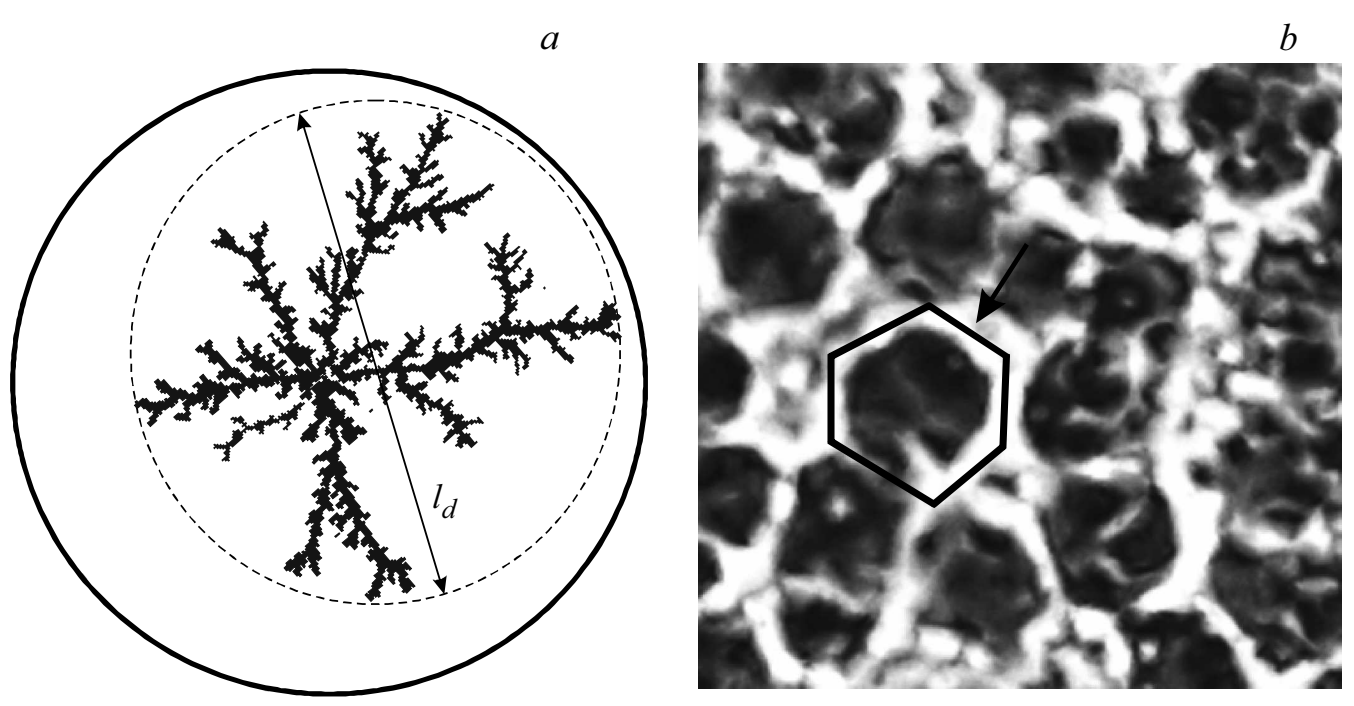

Рис. 3. Компьютерная модель дендрита - кластер с $D_{f} \approx 1.64(a)$ и оптическое изображение ячеистой структуры поверхности тестов $(b)$.

Задача по определению меры $M$ объекта заключается в определении того, сколько раз $N$ измеряемый объект, вложенный в пространство $R^{D}$ с размерностью $D$, может быть заполнен неким измерительным (калибровочным) объектом, описываемым функцией

$$
d(\delta)=\gamma(D) \delta^{D}
$$

(например, отрезком $\left(D=D_{T}=1\right)$, квадратом или кругом $\left(D=D_{T}=2\right)$, кубиком $\left.\left(D=D_{T}=3\right)\right)$. Здесь $D_{T}$ представленная целыми числами топологическая размерность одномерного, двухмерного и трехмерного евклидова пространства, $\gamma$ - нормировочный коэффициент, а $\delta<1-$ безразмерный масштаб. Тогда, согласно [55],

$$
N=\frac{1}{\delta^{D_{\mathrm{H}}}}
$$

где $D_{\mathrm{H}}$ является размерностью пространства Хаусдорфа-Безиковича [55].

В большинстве известных к настоящему времени случаев для определения фрактальных мер $M$ рассматриваются только неотрицательные функции, определенные, как указывалось выше, на семействах множеств пространств Минковского. Например, для измерения линии (одномерный объект) используются отрезки, для измерения площади (двухмерный объект) - квадраты, а для измерения объема (трехмерный объект) - кубы.

Такой подход позволяет исследовать особенности геометрии объекта, но не позволяет установить связь этой геометрии с его (объекта) электрофизическими свойствами.

В качестве множеств пространств Минковского будем рассматривать метрические пространства $R$, образованные парой $R=\{X, \rho\}$, состоящей из некоторого множества $X=\left\{x_{i}\right\}$ элементов $(i=1,2, \ldots, N$ - количество элементов из множества $X)$, в котором определено расстояние $\rho$ между любой парой элементов с аксиомами треугольника, симметрии и нулевого расстояния $[54,56]$.
При этом однозначная, неотрицательная и действительная функция $\rho=\rho(\mathbf{r})$ должна быть определена для любых $\mathbf{r}(\mathbf{r}-$ радиус-вектор между любыми двумя элементами).

Например, метрическое пространство исследуемой тонкопленочной системы $R=\{X, \rho\}$ можно образовать множеством $X$ точек поверхности (рельефа $h(x, y))$ или ее составных элементов: зерен, кристаллитов и диэлектрических промежутков, между любой парой которых определено расстояние $\rho$ с аксиомами треугольника, симметрии и нулевого расстояния.

Между тем известно, что на одном и том же множестве можно задавать разные метрики (меры), описываемые произвольными определенными на этом множестве непрерывными функциями. Множества таких функций образуют функциональные пространства. Для удобства при рассмотрении функциональных пространств часто используют геометрические понятия и определения. Исходя из этого для определения меры можно использовать разные аддитивные функции, если они определены на заданном нормированном линейном пространстве (далее - функциональном пространстве), множество которых удовлетворяет всем аксиомам линейного пространства [57]. Для таких пространств применимы понятия непрерывности и нормы - аналога расстояния между точками в евклидовом пространстве. Под расстоянием между функциями $F_{1}$ и $F_{2}$ из функционального пространства понимают норму $\left\|F_{1}-F_{2}\right\|$. Элементами функционального пространства могут быть произвольные математические объекты: скалярные числа, векторы, матрицы, сингулярные самоподобные (фрактальные) функции и самоподобные (фрактальные) множества [58], что очень удобно при описании различных свойств фрактальных объектов. Например, поверхность можно измерять не только площадью, но и плотностью электрического заряда и т.д. 
Фрактальные функции и порождаемые ими самоподобные меры в настоящее время широко используются в теории сжатия информации и компьютерных изображений [59], теории случайных процессов [60], спектральной теории операторов (обработке сигналов). Например, непериодические и нерегулярные хаотические колебания сигналов описываются теорией детерминированного хаоса и могут быть описаны только на языке фрактальной геометрии [61-63].

Использование самоподобных функций в различных функциональных пространствах при описании процессов в современных электронных (например, полупроводниковых) технологиях крайне ограничено. Причиной этому обычно являются представления о том, что все процессы, происходящие на поверхностях, интерфейсах и в объеме электронных приборов обусловлены однородностью пространств, а имеющиеся неоднородности являются исключением и определяются дефектами, примесями и в некоторых случаях анизотропными свойствами (например, анизотропией свойств кристаллической решетки). На самом деле конструкции полупроводниковых приборов могут обладать и нелинейными свойствами [43-46,64-68].

В общем случае не существует универсальных функциональных пространств, поэтому в зависимости от рассматриваемых задач, в каждом конкретном случае необходимо использовать функциональное пространство, характер которого будет определяться классом используемых функций (видом метрики), описывающих какуюлибо из электрофизических характеристик объекта.

При этом функции, описывающие средние значения сопротивлений $R$, как и множества других электрофизических характеристик в электронных технологиях, не являются фрактальными. Фрактальность появляется только после ввода пространственных координат $(x, y, z)$, что, собственно, и позволяет количественно описать различные размерные эффекты.

Для описания слоевого сопротивления $R_{s q}$ исследуемой тонкопленочной металлической системы в зависимости от координат $(x, y)$ воспользуемся линейным функциональным пространством, состоящим из непрерывных функций $F(x, y, z)$, определенных на некоторой двухмерной области $\Delta r$ евклидова пространства.

В качестве меры $M_{k}=R_{s q}=\Phi$ в таком функциональном пространстве будем рассматривать функционалы $\Phi$ - интегралы от некоторых функций $F(x, y, z)$ по элементам этого пространства:

$$
M_{k}=\Phi=\int_{\Delta r} F(r) d r .
$$

Связь функционального пространства с метрическим можно организовать, поставив в соответствие каждой области из множества метрического пространства $R$ некий функционал $\Phi$ из функционального пространства $\mathbf{M}_{k}-$ меру $M_{k}$, описывающую некую $k$-ую аддитивную (интегральную) электрофизическую характеристику, где $k=1,2, \ldots, K$ количество мер, $\mathbf{M}_{k}=\left\{M_{k, i}\right\}$.
При этом все аргументы функций $F=M_{k}$ линейного пространства $\mathbf{M}_{k}$ должны принадлежать множеству метрического пространства $R$.

Таким образом, каждому $i$-му элементу из множества метрического пространства $R$ можно поставить в соответствие интегральную меру $M_{k, i}$ (функционал) из функционального пространства, описывающую некую $k$-ую аддитивную (интегральную) электрофизическую характеристику, где $k=1,2, \ldots, K-$ количество мер, $\mathbf{M}_{k}=\left\{M_{k, i}\right\}$, а $i=1,2, \ldots, N-$ количество элементов.

Например, в качестве таких интегральных характеристик элементов функционального пространства тонкопленочной системы можно рассматривать пространство сопротивлений $\mathbf{M}_{k=1}=\left\{R_{s q, i}\right\}$, масс $\mathbf{M}_{k=2}=\left\{m_{i}\right\}$, электрических зарядов $\mathbf{M}_{k=3}=\left\{Q_{i}\right\}$ и др.

Объединение $R$ и $\mathbf{M}_{k}$ образует метрическое пространство с мерой $S_{M, k}=\left\{R, \mathbf{M}_{k}\right\}$, представляющее собой совокупность элементов метрического пространства $R$, в зависимость от которого (через аргументы) поставлено в соответствие некоторое множество мер $M_{k, i}$ из линейного функционального пространства $\mathbf{M}_{k}$ [54]. При этом размерность как метрических пространств $R$, так и функциональных пространств $\mathbf{M}_{k}$ может укладываться в топологические размерности евклидовых пространств, так как может быть выражена любым вещественным числом от 0 до 3 .

Например, интегралы от функции плотности электрического заряда, функции плотности электрического тока, или функции плотности массы, можно рассматривать в качестве мер, характеризующих емкостные, проводящие и массогабаритные свойства линейных проводов, двумерных поверхностей или трехмерных объемов. В отличие от метрического пространства $R$ меры (функционалы $\Phi)$ функционального пространства могут иметь и отрицательные значения, как, например, меры электрических зарядов.

Таким образом, множество $X$ тестовых контактов будет принадлежать метрическому пространству $R=\{X, \rho\}$, а множество мер $\left\{M_{k, i}\right\}$ этих тестов, аргументы (переменные) которых определены на множестве $X,-$ функциональным пространствам.

В данных экспериментах $N=5$ (пять тестовых элементов, $i=1, \ldots, 5)$, а $k=1$, так как будем рассматривать только меру $M_{1, i}$ одной электрической характеристики $R_{\mathrm{O}}$. Поэтому далее, для упрощения обозначений, будем полагать $M_{1, i} \equiv M_{i}=R_{\mathrm{O} i}, \mathbf{M}_{1} \equiv \mathbf{M}$ и $S_{M}=\{R, \mathbf{M}\}$.

Для определения хаусдорфовой размерности $D_{\mathrm{H}}$ метрических пространств исследуемых тестов использовался метод, основанный на определении размерности подобия $D_{S}$ путем подсчета относительного увеличения меры объекта $M$ в $\eta=M_{i+1} / M_{i}$ раз при относительном уменьшении линейных размеров измерительного масштаба в $\zeta=l_{i} / l_{i+1}$ раз [55].

$$
D_{S}=\frac{\ln (\eta)}{\ln (\zeta)}=-\frac{\ln (N)}{\ln (\delta)}, \quad \delta=1 / \zeta<1 .
$$


Значения $R_{\mathrm{O}}, D_{S, 1}\left(R_{\mathrm{O}}\right)$ и $D_{S, 2}\left(R_{\mathrm{O}}\right)$ в зависимости от радиуса $r$ тестовых TiAlNiAu-контактов

\begin{tabular}{c|c|c|c|c|c}
\hline & \multicolumn{5}{|c}{$r$, мкм } \\
\cline { 2 - 6 } & 500 & 200 & 100 & 40 & 20 \\
\hline$R_{\mathrm{O}}, \mathrm{OM}_{\mathrm{y}}$ & $2.38 \pm 0.16$ & $1.72 \pm 0.07$ & $1.10 \pm 0.09$ & $0.64 \pm 0.05$ & $0.36 \pm 0.04$ \\
$D_{S, 1}\left(R_{\mathrm{O}}\right)$ & 1.59 & 1.68 & 1.69 & 1.63 & - \\
$D_{S, 2}\left(R_{\mathrm{O}}\right)$ & - & 1.35 & 1.48 & 1.57 & 1.59
\end{tabular}

Для этого осуществлялась трассировка растровых изображений рельефов поверхностей исследуемых тонкопленочных контактов при заданном значении измерительного масштаба $l$ и подсчитывалось получившееся количество замкнутых контуров заполнения. На первой итерации значение измерительного масштаба $l_{0}$ принималось равным радиусу тестового контакта $r$ и подсчитывалось количество $N_{0}$ полученных непересекающихся замкнутых контуров нулевого уровня. На следующем шаге размер $l_{0}$ уменьшался до $l_{1}$ так, чтобы после трассировки получалось целое количество $N_{1}>N_{0}$ контуров, вложенных в контуры первого уровня, и так далее, пока $l_{i}$ не станет равным минимальному расстоянию между пикселями изображения. В качестве меры метрического пространства объекта $M$ выбиралось максимальное количество $N$ вложенных контуров, соответствующих минимальному размеру $l$. Подставив в выражение (5) $\xi=l_{0} / l_{i}$ и $\eta=N_{i}$, определялось значение размерности подобия $D_{S}$ метрического пространства поверхности.

Размерности подобия $D_{S}$ и Хаусдорфа $D_{\mathrm{H}}$ близки по

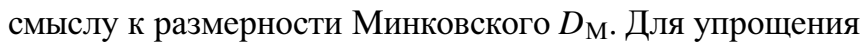
физических представлений ограничимся приближением, когда фрактальная размерность $D_{f}$ равна $D_{\mathrm{M}}$.

Согласно условию Мандельброта, для фрактальных объектов значение $D_{f}$ должно быть больше значения топологической размерности евклидова пространства $D_{f}>D_{\mathrm{T}}$. Например, для линейных объектов $D_{f}>1$, а для двумерных $-D_{f}>2$.

Согласно $[47,48]$, мера фрактального объекта в зависимости от его линейного размера $l$ может быть выражена следующим образом:

$$
M_{i}=M_{0}\left(\frac{l_{i}}{l_{0}}\right)^{2 D_{\mathrm{T}}-D_{f}} .
$$

В случае предельного перехода из локального приближения в глобальное, когда значения $D_{f}$ и $D_{\mathrm{T}}$ совпадают, выражение (6) переходит в известное выражение для объектов с целой топологической размерностью:

$$
M_{i}=M_{0}\left(\frac{l_{i}}{l_{0}}\right)^{D_{\mathrm{T}}} .
$$

Так как элементам рассматриваемого функционального пространства (функционалам) могут быть присущи основные свойства метрических линейных пространств: треугольника, симметрии и нулевого расстояния, то для определения их размерности также может быть использовано выражение (5), где в качестве $M_{0}$ выбиралось исходное (минимальное или максимальное) значение слоевого сопротивления $R_{\mathrm{O}, 1}=0.36$ Ом тестового контакта, а в качестве $l_{0}$ выбиралось значение радиуса этого теста $l_{0}=r_{1}$. Полагая $\eta=M_{i} / M_{0}$, а $\xi=l_{i} / l_{0}$, из (5) можно определить значения $D_{f}$ функциональных пространств слоевых сопротивлений [47].

\section{4. Результаты экспериментов и их обсуждение}

Вопреки общепринятым представлениям, слоевые сопротивления $R_{\mathrm{O} i}$ изготовленных TiAlNiAu тестовых контактов при увеличении их радиуса от $r_{1}=20$ до $r_{5}=500$ мкм увеличиваются более чем в 6 раз, от 0.36 до 2.38 Ом (см. таблицу). Наблюдаемый размерный эффект можно объяснить особенностями их внутреннего строения, так как средний разброс толщин $d=310 \pm 60$ нм не может обеспечить столь большие различия в значениях слоевых сопротивлений $R_{\mathrm{O} i}$. Возможная причина зависимости $R_{\mathrm{O} i}$ от радиуса $r_{i}$ видится во фрактальной структуре этих тестов.

Согласно оптическим изображениям, тесты имеют сильно развитую морфологию поверхности, образованную ячеистой структурой (рис. $1, \mathrm{I}$ ) и разросшимися на ее фоне кристаллитами (дендритами) (рис. 1, II). Размеры ячеек и плотность их заполнения не зависят от радиуса $r$ тестовых контактов и в среднем составляют $4 \pm 0.5$ мкм. Из анализа растровых изображений (рис. $1, \mathrm{I}$ ) следует, что ячеистая структура представляет собой ячейки, напоминающие собой шестигранные ячейки Бенара (рис. 3, $b$, указано стрелкой). Ячейки образованы стенками высотой $\sim 300$ нм и толщиной у основания 30-50 нм. Согласно [53], пространство внутри ячеек и между дендритами сформировано плохо проводящей системой $\mathrm{AuAl}$, а стенки ячеек - из $\mathrm{NiAu}$. Подобные образования возникают в результате двумерной ограниченной диффузией агрегации частиц $[53,55]$.

Картографирование элементного состава тестовых контактов методом энергодисперсионного рентгеноспектрального анализа с использованием растрового электронного микроскопа „Quanta 200 3D“ в целом подтвердило результаты [53] с учетом практически полного отсутствия $\mathrm{Ni}$ на дне ячеек и большего содержания золота на стенках и в дендритах. 
Разросшиеся в двумерной плоскости дендриты выступают над поверхностью ячеек на $\sim 60-100$ нм. Они образованы выпуклостями, сформированными в процессе быстрого термического отжига из-за агрегации относительно хорошо проводящего сплава $\mathrm{NiAu}$ в локальных областях тонкопленочной TiAlNiAu-системы [53].

Из рис. 1, II следует, что средние размеры и количество линейных дендритов на поверхности тестов ступенчато увеличиваются с увеличением радиуса $r$ контактов. Латеральный размер дендрита определялся по радиусу $l_{d}$, описанной вокруг него окружности (рис. 3, $a$, пунктир). Согласно рис. 1, II, на наименьшем контакте, радиусом $r_{1}=20$ мкм, располагается только часть дендрита, а остальная часть поверхности теста образована ячеистой структурой, подробно изображенной на pис. $3, b$. На контакте радиусом $r_{2}=40$ мкм уже может разместиться целый дендрит размерами, достигающими $<30$ мкм (рис. $1, b)$. Увеличение радиуса $r_{3}$ до 100 мкм приводит к увеличению количества дендритов на поверхности тестовых элементов до 4-6 штук при сохранении их средних размеров $l_{d}<30$ мкм (рис. $1, c$ ). Дальнейшее увеличение радиуса $r_{4}$ до 200 мкм приводит к росту линейных размеров дендритов до $l_{d}<90$ мкм при сохранении их среднего количества (4-6 штук) на поверхности теста (рис. $1, d$ ). Последующее увеличение радиуса $r_{5}$ до 500 мкм сопровождается дальнейшим увеличением количества дендритов на поверхности тестов до 12-15 штук при сохранении их средних размеров $l_{d}<90$ мкм (рис. $1, e$ ). Непропорциональные скачкообразные изменения размеров дендритов и их количества, происходящие при увеличении линейных размеров тестов $r$, могут указывать на наличие у них субструктуры мультифрактальной геометрии (по аналогии с „чертовой“ лестницей).

Определенные с использованием выражений (5) значения хаусдорфовой размерности $D_{\mathrm{H}}$ метрических пространств поверхностей тестов имеют дробные значения, характерные для фрактальных объектов с дробной фрактальной размерностью $D_{f}$. При этом увеличение радиуса контактов приводит и к увеличению значений $D_{f}$. Так, для $r_{1}=20$ мкм значение $D_{f}=2.04$, для $r_{2}=40$ мкм $-D_{f}=2.06$, для $r_{3}=100$ мкм $D_{f}=2.38$, для $r_{4}=200$ мкм $-D_{f}=2.40$ и для $r_{5}=500$ мкм - $D_{f}=2.54$.

Близкие к топологической размерности $D_{\mathrm{T}}=2$ значения $D_{f}$ контактов малого $\left(r_{1}=20, r_{2}=40\right.$ мкм $)$ радиуса указывают на то, что основную роль в формировании их рельефа играет ячеистая структура. Резкое увеличение значений $D_{f}$ для контактов с радиусами, превышающими 100 мкм, указывает на заметное влияние дендритов на формирование рельефа поверхности таких тестов. Полученные значения $2<D_{f}<3$ указывают на то, что помимо двумерных свойств поверхностям тестов могут быть присущи и некоторые объемные свойства [55].

Исследование контурного изображения, полученного методом трассировки оптических изображений поверхности (рис. 1, II), позволило определить среднее значение фрактальной размерности $D_{f}$ линейных дендритов
$D_{f} \approx 1.66$ (5). Полученное значение $1<D_{f}<2$ указывает на то, что из-за разветвленности в плоскости контакта линейным дендритам присущи и некоторые свойства двумерных объектов [55]. На рис. 3, а представлена компьютерная модель подобного дендрита с фрактальной размерностью $D_{f}=1.63$, значение которой хорошо совпадает с экспериментально определенным значением $D_{f} \approx 1.66$.

Согласно (6), а также [43,44,47], зависимость слоевого сопротивления тестов $R_{s q}$ от их линейных размеров может быть представлена следующими степенными зависимостями:

$$
R_{\square, i}=R_{\square, 0}\left(\frac{a_{0}}{a_{i}}\right)^{D_{\mathrm{T}}-D_{f}} \text { и } R_{\mathrm{O}, i}=R_{\mathrm{O}, 0}\left(\frac{r_{0}}{r_{i}}\right)^{D_{\mathrm{T}}-D_{f}} .
$$

Выражения (8) описывают также и случай предельного перехода из локального приближения в глобальное, когда значения $D_{f}$ и $D_{\mathrm{T}}$ совпадают, и $R_{s q}$ перестает зависеть от линейных размеров измеряемых участков: $R_{\square, i}=R_{\square, 0}$ и $R_{\mathrm{O}, i}=R_{\mathrm{O}, 0}$.

Фрактальный дендрит отличается от обычного тем, что с увеличением расстояния от центра его плотность убывает по степенному закону [55]. Степенью в этом случае может служить размерность подобия $D_{S}$, или фрактальная размерность $D_{f}(8)$, которые являются количественными характеристиками того, насколько неравномерно кристаллит заполняет занимаемое им на плоскости топологическое пространство [55]. Уменьшение плотности фрактального кристаллита с увеличением расстояния от его центра вызвано увеличивающейся разветвленностью в пространстве его составных частей, что, согласно их эквивалентной электрической схеме, будет сопровождаться и ростом результирующего электрического сопротивления. При этом увеличение разветвленности кристаллита сопровождается увеличением протяженности его границ, а следовательно, и увеличением протяженности диэлектрических промежутков между соседними кристаллитами. Это приводит к дополнительному рассеянию электронов проводимости на таких границах и, согласно модели проводимости Маядаса-Шатцкеса, к увеличению слоевого сопротивления тестов при увеличении их линейных размеров, что мы и наблюдаем в экспериментах.

В таблице представлены значения размерностей функционального пространства $D_{S}\left(R_{\mathrm{O}}\right)$ для всех пяти тестов. Видно, что определенные из (5) средние значения $D_{S}\left(R_{\mathrm{O}}\right)$ имеют дробные значения, близкие к среднему значению $D_{S}$ метрического пространства кристаллитов. Совпадение значений $D_{f}$ кристаллитов со значением $D_{f}$ функционального пространства слоевых сопротивлений $R_{\square((\mathrm{O})}$ указывает на то, что основную проводимость тонкопленочной системы тестовых контактов обеспечивают

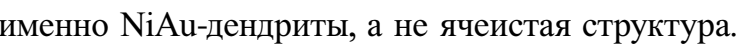

Таким образом, можно заключить, что латеральная проводимость тестов в основном обеспечивается кристаллитами, образованными агрегацией из ограниченной диффузии сплава $\mathrm{NiAu}$ [53], обладающего значительно лучшей проводимостью, чем заполняющий ячеистую структуру сплав $\mathrm{AlAu}$. 
Полученные экспериментальные результаты указывают на то, что самоподобному метрическому пространству $R$ можно подобрать такое определенное на $R$ функциональное пространство, элементам (функционалам) которого также будут присущи эффекты самоподобия. Это обстоятельство и позволяет объяснить наблюдаемые размерные эффекты, заключающиеся в зависимости электрофизических характеристик (мер $M$ ) объектов от их линейных размеров $[43,47]$.

Согласно представленным в таблице результатам, значение хаусдорфовой размерности функционального пространства $D_{\mathrm{H}}$ относительно и само зависит от выбора начала отсчета (системы координат или точки зрения наблюдателя), что согласуется с одним из признаков хаотических систем. В случае, когда в качестве $M_{0}$ выбрано минимальное значение $R_{\mathrm{O}, 0}=0.36$ Ом, получаются одни значения $D_{S, 1}\left(R_{\mathrm{O}}\right)$, а в случае, когда в качестве $M_{0}$ выбрано максимальное значение $R_{\mathrm{O}, 0}=2.38 \mathrm{OM}$, - другие значения $D_{S, 2}\left(R_{\mathrm{O}}\right)$ (см. таблицу). Согласно полученным результатам, такая относительность значений $D_{S}$ не создает противоречий в определении значений мер $M$ измеряемых объектов, так как, согласно (6), значение $M$ инвариантно относительно выбора значений $M_{0}, l_{0}$ и $D_{f}$.

Согласно дополнительным наблюдениям, в зависимости от конструкции полупроводниковой структуры, толщин металлических слоев, способов их нанесения и формирования размеры и поверхностная плотность дендритов и ячеек могут меняться, но в целом принципы формирования TiAlNiAu-металлизации остаются одними и теми же.

\section{5. Заключение}

Полученные экспериментальные результаты позволили выявить в локальном приближении связь геометрии метрического пространства тонкопленочной металлической TiAlNiAu-системы с геометрией функционального пространства ее слоевых сопротивлений $R_{s q}$. Это позволило объяснить природу наблюдаемых размерных эффектов, заключающихся в зависимости слоевых сопротивлений $R_{s q}$ тонкопленочных металлических систем на основе TiAlNiAu от их латеральных (в плоскости $(x, y)$ ) линейных размеров. Значение фрактальной размерности $D_{f}$ образующих ее $\mathrm{NiAu}$-дендритов определяет зависимость $R_{s q}$ от линейных размеров. Обнаруженная закономерность имеет важное практическое значение как для точного расчета значений $R_{s q}$ тонкопленочных металлических систем при проектировании омических и барьерных контактов, тонкопленочных резисторов, конденсаторов, линий СВЧ-передач, контактных площадок, так и для контроля результатов технологических процессов получения тонких металлических пленок и систем на их основе на микронном и наноуровнях.

Работа выполнена при финансовой поддержке проекта „Исследования и разработки технологии изготовления сверхвысокочастотных монолитных интегральных схем на основе гетероструктур InAlN/GaN для изделий кос- мического применения“ (Соглашение № 14.578.21.0240 от 26.09.2017 г.) УИР RFMEFI 57817Х240.

\section{Список литературы}

[1] A.F. Mayadas, M. Shatzkes, J.F. Janak. Appl. Phys. Lett., $14(11), 345$ (1969).

[2] Г. Хасс. Физика тонких пленок (М., Мир, 1967) т. 1.

[3] Р.Э. Тун. Структура тонких пленок. В сб.: Физика тонких пленок (М., Мир, 1967) т. 1. [Пер. с англ. под ред. Г. Хасса].

[4] К.Л. Чопра. Электрические явления в тонких пленках (М., Мир, 1972).

[5] Ю.Ф. Комник. Физика металлических пленок. Размерные и структурные эфбекты (М., Атомиздат, 1979).

[6] Д.К. Ларсон. Размерные эфббекты в электропроводности тонких металлических пленок и проволок В кн.: Физика тонких пленок (М., Мир, 1973) т. 6, с. 97.

[7] Н.Т. Гладких. Автореф. докт. дис. (Харьков, 1976).

[8] R. Wu, L. Chen, A.J. Freeman. JMMM, 170, 103 (1997).

[9] Ю.Ю. Тарасевич. Перколяция: теория, приложения, алгоритмы. Учеб. пособие (М., Едиториал УРСС, 2002).

[10] В.Б. Лобода. Автореф. конд. дис. (Ин-т проблем матриаловедения, Киев, 1984).

[11] И.В. Антонец. Автореф. канд. дис. (Сыктывкарский гос. ун-т, Челябинск, 2004).

[12] О.Н. Соклакова. Альтернативная энергетика и экология, 11 (133), 25 (2013).

[13] M.S.P. Lucas. J. Appl. Phys., 36 (5), 1632 (1965).

[14] A. Bendavid, P.J. Martin, L. Wieczorek. Thin Sol. Films, 354, 169 (1999).

[15] G. Bader, A. Haché, V.-V. Truong. Thin Sol. Films, 375, 73 (2000).

[16] И.Е. Проценко, А.В. Яременко. Металлофизика, 4 (2), 71 (1982).

[17] Н.Н. Коненкова, Э.М. Рейхрудель, Г.В. Смирницкая. Микроэлектроника, $9(5), 467$ (1980).

[18] M.S. Iqbal, Ijaz-ur-Rehman, Beg S.J. Pure. Appl. Phys., 18 (8), 614 (1980).

[19] A. Kawazu, Y. Saito, H. Asahi, G. Tominada. Thin Sol. Films, 37 (2), 261 (1976).

[20] Р.В. Бычковский, Е.А. Колесник, Г.А. Ухлинов и др. Размерный эффбект и температурный коэффициент сопротивления тонких пленок висмута В кн.: Сб. науч. тр. по проблемам микроэлектроники (Изд-во МФТИ, 1978) т. 38 , с. 224.

[21] Б.Л. Мельничук, З.В. Стасюк. ФММ, 46 (5), 1115 (1978).

[22] W.E.I. Neal, R.N.A. Borabin. Thin Sol. Films, 44 (1), 169 (1977).

[23] H.-D. Liu, Y.-P. Zhao, G. Ramanath, S.P. Hurerka, G.C. Wang. Thin Sol. Films, 384, 151 (2001).

[24] Kh.M. Mannan. Phys. Lett., 62 (7), 519 (1977).

[25] C.R. Tellier. Vacuum, 28 (8-9), 321 (1978).

[26] A.K. Pal, S.J. Chaudhuri. Mater. Sci., 11 (7), 872 (1976).

[27] Г.И. Фролов, В.С. Жигалов, А.И. Польский, В.Г. Поздняков. ФTT, 38 (4), 1208 (1996).

[28] A.K. Pal, S. Chaudhuri, A.K. Barua. J. Phys. D: Appl. Phys., 9(15), 2261 (1976).

[29] G. Fischer, H. Hoffmann, I. Vancea. Phys. Rev. B: Condens. Matter, 22 (12), 6065 (1980).

[30] K.B. Singh, I. Hatibarua. Indian J. Pure Appl. Phys., 20 (3), 183 (1982).

[31] C.R. Pichard, Yu.P. Komnik, A.I. Tosser. J. Mater. Sci. Lett., 2 (7), 360 (1983). 
[32] А.А Замешин, М.Ю. Попов, В.В. Медведев, С.А. Перфилов, Р.Л. Ломакин, С.Г. Буга, В.Н. Денисов, А.Н. Кириченко, Е.В. Татьянин, В.В. Аксененков, В.Д. Бланк. Тр. МФТИ, 4 (3), 74 (2012).

[33] S.C. Bandyopadhyay, A.K. Pal. J. Phys. D: Appl. Phys., 12 (6), 953 (1979).

[34] В.Б. Лобода, И.Е. Проценко, М.Д. Смолин. Металлофизика, 5 (5), 69 (1983).

[35] M.A. Angadi, L.A. Udachan. Thin Sol. Films, 79 (2), 149 (1981).

[36] Д.К. Ларсон. Физика тонких пленок (М., Мир, 1973) т. 6 , c. 97.

[37] A.D. Kent, J. Yu, U. Rüdiger, S.S.P. Parkin. J. Phys.: Condens. Matter, 13, R461 (2001).

[38] Г.П. Жигальский. УФН, 6, 623 (1997).

[39] И.В. Антонец, О.Н. Котов, С.В. Некипелов, Е.А. Голубев. ЖТФ, 3, 24 (2004).

[40] И.В. Антонец. Тез. докл. VI ВНКСФ (Томск, Россия, 2000) c. 403.

[41] И.В. Антонец. Сб. научных работ аспирантов и молодых ученых (Сыктывкар, 2002) с. 6.

[42] I. Mojzes, C. Dominkovics, G. Harsányi, S. Nagy, J. Pipek, L. Dobos. Appl. Phys. Lett., 91, 073107 (2007).

[43] Н.A. Торхов. Матер. 25-й Междунар. Крымской конф. „СВЧ-техника и телекоммуникационные технологии“ (КрыМиКо-2015) (Севастополь, Крым, Россия, 6-12 сентября 2015) с. 637.

[44] Н.А. Торхов. Тез. докл. 7-й Междунар. науч.-практ. конф. по физике и технологии наногетероструктурной СВЧэлектроники „Мокеровские чтения“ (М., Россия, Нац. исслед. ядерный ун-т „МИФИ“, 2016) с. 51.

[45] Н.А. Торхов, В.А. Новиков. ФТП, 43 (8), 1109 (2009).

[46] Н.А. Торхов, И.Д. Филимонова, В.А. Новиков. Тез. докл. 7-й Междунар. науч.-практич. конф. по физике и технологии наногетероструктурной СВЧ-электроники „Мокеровские чтения“ (М., Россия, Нац. исслед. ядерный ун-т „МИФИ“, 2016) c. 49.

[47] N.A. Torkhov, L.I. Babak, A.A. Kokolov, A.S. Salnikov, I.M. Dobush, V.A. Novikov, I.V. Ivonin. J. Appl. Phys., 119, 094505 (2016)

[48] Н.А. Торхов. Поверхность. Рентгеновские, синхротронные и нейтронные исследования, 1, 1 (2010).

[49] N.M. Shmidt, V.V. Emtsev, A.G. Kolmakov, A.G. Kryzhanovsky, W.V. Lundin, D.S. Poloskin, V.V. Ratnikov, A.N. Titkov, E.E. Zavarin. Nanotechnology, 12, 471 (2001).

[50] N.M. Shmidt, G. Aliev, A.N. Besyul'kin, J. Davies, M.S. Dunaevsky, A.G. Kolmakov, A.V. Loskutov, W.V. Lundin, A.V. Sakharov, A.S. Usikov, D. Wolverson, E.E. Zavarin. Physica Status Solidi C, 558 (2002).

[51] G. Greco, F. Iucolano, F. Roccaforte. Appl. Surf. Sci., 383 (15), 324 (2016).

[52] B. Van Daele, G. Van Tendeloo, W. Ruythooren, J. Derluyn, M.R. Leys, M. Germain. Appl. Phys. Lett., 87, 061905 (2005).

[53] R. Gong, J. Wang, S. Liu, Z. Dong, M. Yu, C.P. Wen, Y. Cai, B. Zhang. Appl. Phys. Lett., 97, 062115 (2010).

[54] А.Н. Колмогоров, С.В. Фомин. Элементы теории функций и функционального анализа. Изд. 4-е перераб. (М., Наука, 1976) с. 543.

[55] Е. Федер. Фракталы (М., Мир, 1991).

[56] И.А. Иванишко, В.Г. Кротов. Мат. заметки, 86 (6), 829 (2009).

[57] И.М. Гельфанд, С.В. Фомин. Вариационное исчисление (М., Гос. изд-во физ.-мат. лит., В-71, 1961).
[58] Ю.В. Тихонов. Автореф. канд. дис. (М., МГУ им. М.В. Ломоносова, 2016).

[59] M. Barnsley, Fractals everywhere. (U.K., Academic Press Ltd, 1988) p. 533.

[60] А.И. Назаров. Вероятность и статистика. ЗНС ПОМИ, 311, 190 (2004).

[61] K. Aoki. Nonlinear Dynamics Chaos in Semiconductors (U.K., British Library Cataloguing-in-Publication Data. IOP Publishing Ltd, 2001) p. 508

[62] A.P. Micolich. Fractal Magnetic-conductance Fluctuations in Mesoscopic Semiconductor Billiards, Ph.D. Thesis (University of New South Wales. U.K., 2000).

[63] A.P. Micolich, R.P. Taylor, A.G. Davies, T.M. Fromhold, H. Linke, L.D. Macks, R. Newbury, A. Ehlert, W.R. Tribe, E.H. Linfield, D.A. Ritchie. Appl. Phys. Lett., 80, 4381 (2002).

[64] Н.А. Торхов. Матер. 25-й Междунар. Крымской конф. „СВЧ-техника и телекоммуникационные технологии“ (КрыМиКо-2014) (Севастополь, Крым, Россия, 7-13 сентября 2014) с. 663.

[65] R. Taylor, R. Newbury, A. Micolich, M. Fromhold, H. Linke, G. Davies, T. Martin, C. Marlow. A Review of Fractal Conductance Fluctuations in Ballistic Semiconductor Devices (2003) p. 277.

[66] Н.А. Торхов, В.Г. Божков, В.А. Новиков, Н.В. Земскова, В.А Бурмистрова. Поверхность. Рентгеновские, синхротронные и нейтронные исследования, 1, 81 (2011).

[67] Н.А. Торхов, В.Г. Божков. ФТП, 43 (5), 577 (2009).

[68] Н.А. Торхов, В.А. Новиков, И.В. Ивонин. Матер. 25-й Междунар. Крымской конф. „СВЧ-техника и телекоммуникационные технологии“ (КрыМиКо-2015) (Севастополь, Крым, Россия, 6-12 сентября 2015) с. 609.

Редактор Г.А. Оганесян

\section{Sheet Resistance of TiAINiAu thin film metallization of ohmic contacts to nitride semiconductor structures}

\author{
N.A. Torkhov ${ }^{1,2,3}$ \\ ${ }^{1}$ Scientific-Research Institute of Semiconductors, \\ 634034 Tomsk, Russia \\ 2 Tomsk State University, \\ 634050 Tomsk, Russia \\ 3 Tomsk State University of Control System \\ and Redioelectronics, \\ 634050 Tomsk, Russia
}

Abstract This paper determines the relation between the geometry of metric space of TiAlNiAu thin film metal system surface and the geometry of functional space of its sheet resistances $R_{s q}$. The investigation provides a means to describe a lateral size effect observed in local approximation that involves a dependency of sheet resistance $R_{s q}$ of TiAlNiAu metal film on its lateral linear dimensions (in $(x, y)$ plane). This dependency is defined by fractal geometry of dendrites, or, more specifically, it is a power-law dependency of linear dimension variation on fractal dimension $D_{f}$. The revealed relation has a great practical importance both for a precise calculation of thin film metal system $R_{s q}$ values in designing discreet devices and ICs, and for controlling results at micro- and nanoscale in producing workflow for thin metal films and systems based on them. 